NASA CONTRACTOR REPOR T

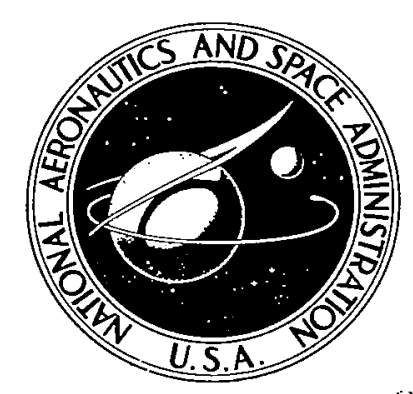

NASA CR-11

LCAN COPY: RETURN TO AFWL (WLIL-2)

KIRTLANB AFB; NATEX

\title{
MATHEMATICAL PROBLEMS \\ OF MODELING STOCHASTIC \\ NONLINEAR DYNAMIC SYSTEMS
}

\section{by Richard E. Mortensen}

Prepared by

TRW SYSTEMS GROUP

Redondo Beach, Calif.

for Ames Research Center

NATIONAL AERONAUTICS AND SPACE ADMINISTRATION - WASHINGTON, D. C. • SEPTEMBER 1968 

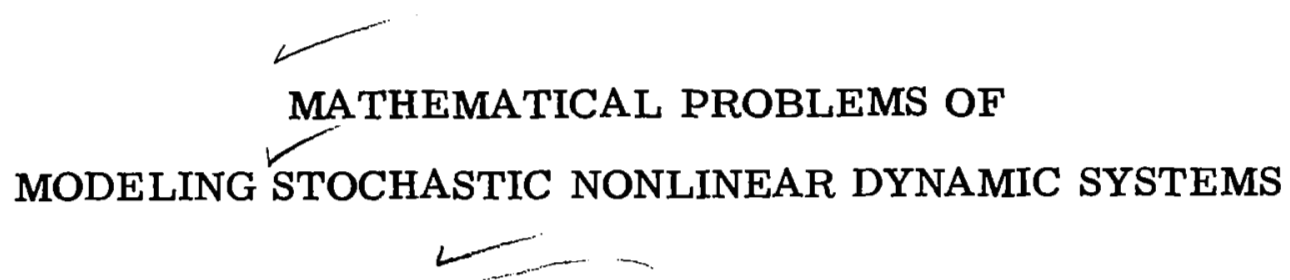

By Richard E. Mortensen

Distribution of this report is provided in the interest of information exchange. Responsibility for the contents resides in the author or organization that prepared it.

Prepared ynder Contract No. NAS 2-4553 by

TRW SYSTEMS GROUP

Redondo Beach, Calif.

for Ames Research Center

NATIONAL AERONAUTICS AND SPACE ADMINISTRATION

For sale by the Clearinghouse for Federal Scientific and Technical Information Springfield, Virginia 22151 - CFSTI price $\$ 3.00$ 



\section{CONTENTS}

\section{Page}

SUMMARY . . . . . . . . . . . . . . . i

I. INTRODUCTION ................. . 2

II. SYMBOLS . . . . . . . . . . . . . . 4

III. THE ENGINEERING MODEL . . . . . . . . . . . . . 7

IV. THE FOKKER-PLANCK EQUATION . . . . . . . . . 9

V. AN APPARENT PARADOX .............. 14

VI. THE ITO CALCULUS .. . . . . . . . . . 20

VII. THE STRATONOVICH CALCULUS . . . . . . . . . 31

VIII. MODELING THE REAL WORLD . . . . . . . . . . 36

IX. CONCLUSION ............... 48

X. REFERENCES . . . . . . . . . . . . . 49 


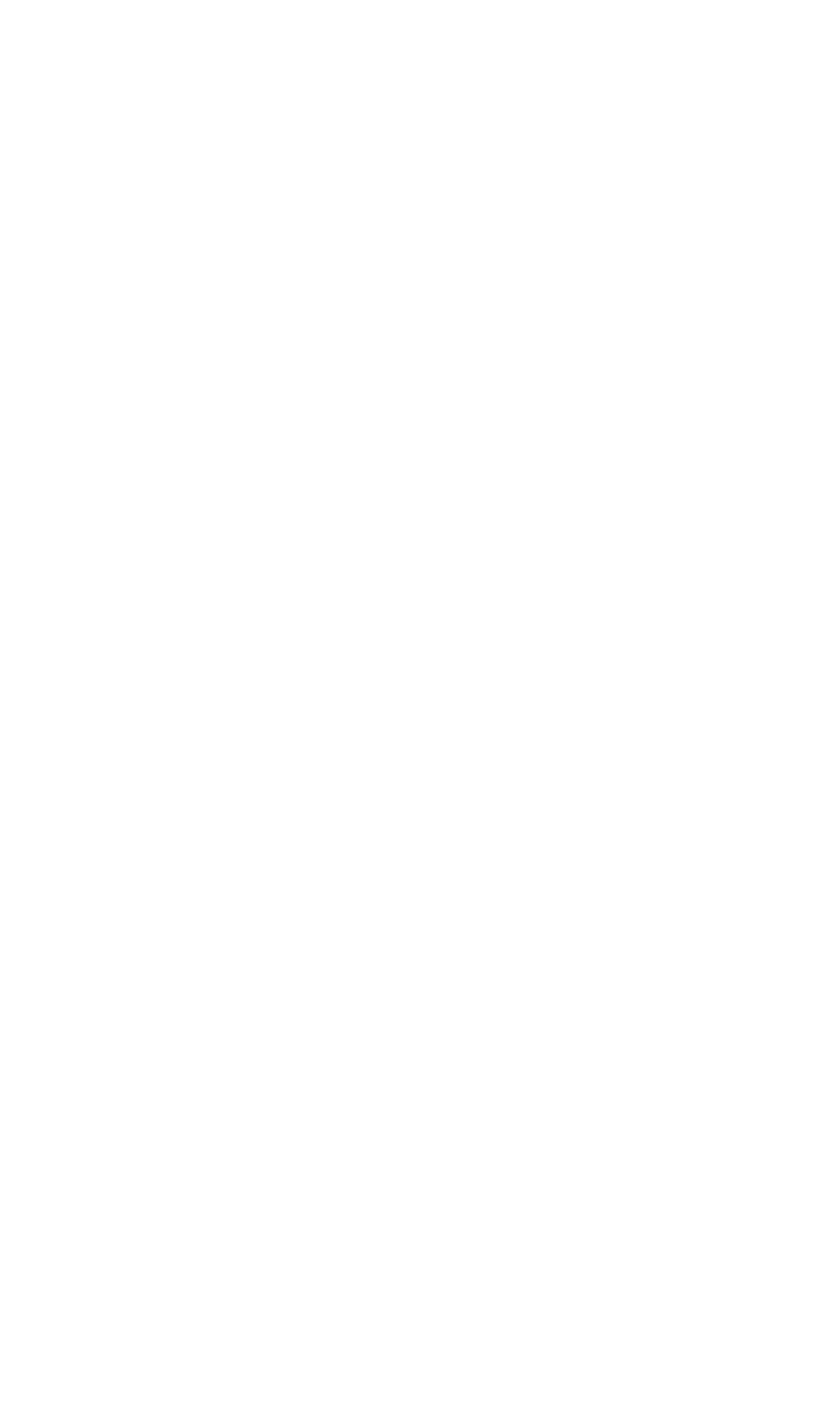




\section{MATHEMATICAL PROBLEMS OF \\ MODELING STOCHASTIC NONLINEAR DYNAMIC SYSTEMS \\ By Richard E. Mortensen \\ TRW Systems Group}

ABSTRACT

The purpose of this report is to introduce the engineer to the area of stochastic differential equations, and to make him aware of some of the mathematical techniques and pitfalls in this area. Topics discussed include continuous-time Markov processes, the Fokker-Planck-Kolmogorov equations, the Ito and Stratonovich stochastic calculi, and the problem of modeling physical systems. 


\section{MATHEMATICAL PROBLEMS OF \\ MODELING STOCHASTIC NONLINEAR DYNAMIC SYSTEMS \\ By Richard E. Mortensen* \\ TRW Systems Group}

\section{SUMMARY}

Certain mathematical problems arise when one attempts to model a stochastic dynamic system by means of a set of nonlinear ordinary differential equations with white noise excitation. This report reviews the basic facts about Markov processes and the Fokker-Planck-Kolmogorov equations, and then illustrates the reason for the difficulties by an example. Two current methods of treating stochastic differential equations, the Ito method and the Stratonovich method, are defined and discussed. It is shown that each method is consistent within itself, that, if properly used, the two methods are equivalent, and finally it is shown how to translate results from one method to the other. A philosophy for modeling physical systems with stochastic differential equations is then advocated. The purpose of this report is to introduce the engineer to the area of stochastic differential equations, and make him aware of some of the mathematical techniques and pitfalls in this area.

* Assistant Professor of Engineering, University of California at Los Angeles, Los Angeles, California. (Consultant at TRW Systems Group) 


\section{INTRODUCTION}

This paper discusses certain mathematical problems which arise in attempting to model a stochastic dynamic system by means of a set of nonlinear ordinary differential equations with white noise excitation. This approach has been advocated in engineering literature at various times over the past ten years. The appeal of this approach is that it is the natural extension to stochastic systems of the state space approach to deterministic systems which has met so much success in optimal control theory. Furthermore, the state vector in such a model turns out to be a vector Markov process, for which a substantial mathematical theory exists; in particular, there is the theory of the Kolmogorov or FokkerPlanck partial differential equation. In addition, as one would expect of a state space approach, this method is especially suited to the study of the transient behavior of the stochastic system, with steady-state, or more precisely, stationary behavior obtained as a limiting case.

The engineering literature tends to give one the impression that the major difficulties associated with this approach are computational. Although it is not denied that the computational difficulties are large, it is the main point of this paper to show that a fundamental difficulty may arise at an earlier phase of the analysis, namely, when the mathematical model itself is chosen. In a sense, this difficulty is not computational but conceptual, i.e. there may be a basic divergence between the implications of the mathematical model and the facts of physical reality.

This difficulty arises from the properties of the heuristic mathematical idealization known as white noise, or its rigorous counterpart Brownian motion, which is heuristically the time integral of white noise. The peculiar implications of the Brownian motion stochastic process puzzled physicists of an earlier era, leading them to adopt a stochastic process with more "physical" properties, the OrnsteinUhlenbeck stochastic process. 
Mathematically, the trouble arises when one attempts to apply the usual rules of differential and integral calculus to functions of time which are actually sample functions of a stochastic process. The result has been that something of a controversy has appeared in recent literature concerning two possible ways of extending ordinary calculus to stochastic functions: the somcalled stratonovich calculus, in which the usual rules continue to apply, and the so-called Ito calculus, in which the rules are changed. Although this subject has been discussed in several papers in the last two or three years, one gets the impression after reading some of these papers that the subject is more bewildering to the reader than it was before he read the paper.

The aim of this report is to show, by means of examples which we have attempted to choose to be as lucid as possible, the reasons for this divergence. Further, we will suggest an approach to the problems of mathematical modeling, analysis, and computation which seems to have the qualities of being both mathematically rigorous and consistent with physical reality. 


\section{SYMBOIS}

A

a

B

$\mathrm{C}$

$\mathrm{d}_{I} \mathrm{~F}$

$d t$

$d x$

$d w$

E

e

F

f

G

g

$\mathrm{h}$

$I_{n}$

(I)

i

$J$

$\mathrm{K}_{1}$

$\mathrm{K}_{2}$

k

m

$\min$ plant matrix

acceleration, $\mathrm{ft} / \mathrm{sec}^{2}$

input matrix

covariance matrix

Ito differential of $\mathrm{F}$

time differential

differential of $x$

differential of Wiener process

expectation operator

base of natural logarithms

force, pounds

vector valued function

matrix valued function

time-varying coefficient

a function

approximation to an Ito integral

Ito integral

an index

an Ito integral

a constant

Lipschitz constant

an integer

mass, slugs

minimum of 
n

$n_{x}$

$\mathrm{n}$

n)

n

$o(\Delta t)$

$\mathrm{p}_{\Delta \mathrm{w}}$

$p(\xi, t)$

$p(\xi, t \mid \eta, s)$

$q(\xi, t)$

$q(\xi, t \mid \eta, s)$

$\mathbf{s}$

(S)

t

V

$v(t)$

$v^{\prime}(t)$

$\mathbf{v}_{\mathrm{X}}$

$\mathrm{y}$

W

$x$

$\dot{x}$

$x_{I}$

$x_{s}$

$\mathrm{g}$

$z(t)$ an integer

horizontal random force component

vertical random force component

random force parallel to flight path

random force perpendicular to flight path

a remainder

probability density for $\Delta w$

probability density function

transition density

density for Wiener process

transition density of Wiener process

dummy time variable

Stratonovich integral

time

magnitude of velocity vector

a random process

transpose of $v$

horizontal component of velocity

vertical component of velocity

Wiener process

state vector

time derivative of $x$

Ito solution

Stratonovich solution

a random process

a random process 
$\beta$

$\Delta \mathrm{t}$

$\Delta w(t)$

$\delta$

$\zeta$

$\xi$

$\rho_{I}(\xi, t \mid \eta, s)$ transition density for $x_{I}$

$\rho_{s}(\xi, t \mid \eta, s)$ transition density for $x_{s}$

$\tau$ flight path angle

a time increment

increment of Wiener process

Dirac delta function

dummy variable

dummy variable

dummy time variable 


\section{THE ENGINEERING MODEL}

Typically, the dynamic equations of motion that arise in the analysis of engineering systems are astatement of Newton's law of motion, $F=$ ma, possibly augmented by the inclusion of known frictional or dissipative forces. Although the direct application of $F=$ ma yields second order differential equations, it is well known that it is always possible, by adding more variables, to convert these to a set of coupled, first order, and often nonlinear, differential equations of the form

$$
\dot{x}(t)=f(x(t), t)
$$

Here $x$ and $f$ are $n$-vectors. The vector $x(t)$ is called the state of the system at time $t$.

If now an engineer wishes to modify equation (I) to try to take account of random forces in the environment, a natural way to proceed is to write

$$
\dot{x}(t)=f(x(t), t)+G(x(t), t) v(t)
$$

Here $v(t)$ is an m-vector, representing the random force at time $t$, and $G(x(t), t)$ is an nxm matrix. It is allowed to be a function of $x$ and $t$ to take into account the possibility that the influence of the noise may depend on the state of the system.

The function $v(t)$ is a random process, $i . e$. for each fixed $t$ the value of the function $v(t)$ is a random variable. In the absence of any special knowledge about the nature of the random force, a commonly made assumption is that $\mathrm{v}(t)$ is a so-called Gaussian white noise random process. This means that for each fixed $t$ the random variable has a Gaussian distribution with zero mean and infinite variance. Furthermore, for any two times $t_{1}, t_{2}$, with $t_{1} \neq t_{2}$, the two random variables $v\left(t_{1}\right)$ and $v\left(t_{2}\right)$ are completely independent of each other.

Let $E$ denote expectation, i.e. averaging across the statistical ensemble. Let a prime denote the transpose of a vector or a matrix. Since $v(t)$ is 
a column vector, $\mathrm{V}^{\prime}(t)$ is a row vector. Mathematically, white noise is characterized by the conditions

$$
E\{v(t)\} \equiv 0 ; E\left\{v\left(t_{1}\right) v^{\prime}\left(t_{2}\right)\right\}=c\left(t_{1}\right) \delta\left(t_{1}-t_{2}\right)
$$

Here $c\left(t_{l}\right)$ is an $m \times m$ matrix, called the white noise covariance matrix, which expresses how the components of the vector $v\left(t_{l}\right)$ are correlated among themselves. It is meaningful to speak of such correlation even though each component has infinite variance.

In the case of stationary white noise, the matrix $C$ is constant, independent of time. Strictly, it is only in this case that the name "white" can be justified, because only in this case can one define a power spectral density function. In this case, the power spectral density function is constant, independent of frequency, analogous to the spectrum of white light.

White noise is much the same kind of mathematical pathology in the theory of random processes that the Dirac delta function is in the theory of deterministic functions.

As is by now well appreciated, so long as one does only linear operations on a delta function, it is usually possible to interpret the result in a meaningful way. However, one runs into trouble in trying to do nonlinear things to a delta function. The square or the logarithm of a delta function is meaningless, for example.

A similar situation exists in the case of white noise. If the differential equation (2) is Iinear, i.e. of the form

$$
\dot{x}(t)=A(t) x(t)+B(t) v(t),
$$

then it turns out that there is no difficulty in interpreting what is meant by a solution of this differential equation. As a function of $t, x(t)$ turns out to be a Gaussian random process, and there is no controversy about how to compute the mean and the covariance of this 
process. The process $x(t)$ is much better behaved than $v(t)$, e.g. none of the components of $x(t)$ has infinite variance.

However, when the differential equation (2) is nonlinear, a problem of interpretation arises. One might at first think that the nonlinear equation (2) is simply meaningless, as in the case of the square of a delta function. However, this is not the case. It turns out that there are two distinct, meaningful ways of interpreting equation (2) which appear in contemporary literature, and which are called respectively the Ito and the Stratonovich interpretations.

As stated in the Introduction, this report will explore this ItoStratonovich divergence. Each interpretation will be explained, as well as the reason for the divergence. The two interpretations will be shown to be equivalent, in the sense that it is possible to pass from the results obtained under one interpretation to the results for the other interpretation via a transformation formula. Finally, the problems of real world modelling and computation will be discussed.

IV. THE FOKKER-PLANCK EQUATION

Before discussing this divergence and the subtleties of the stochastic calculus, perhaps it will be well to review the area of the theory in which there is no controversy. For ease of exposition, henceforth we will consider only scalar-valued random processes, although the theory holds in the vector valued case also.

Engineers can find an introduction to the theory of the Fokker-Planck equation in references 1 and 2 , which also contain further references. This theory will not be developed here, but the major results will be stated.

Consider the scalar stochastic differential equation

$$
\dot{x}(t)=f(x(t), t)+g(t) v(t)
$$


Here $v(t)$ is Gaussian white noise, with

$$
E\{v(t)\}=0 ; E\{v(t) v(s)\}=\delta(t-s)
$$

We assume that $|g(t)|>0$ for all $t$. We will also assume that $f(x, t)$ and $g(t)$ are at least piecewise continuous functions of $t$, that $f$ is at least once differentiable with respect to $x$, and that $f$ obeys the following condition: there exist $K_{1}, K_{2}<\infty$ such that

$$
|f(x, t)| \leq K_{1}+K_{2}|x|
$$

for all $t$ and all $x$.

Aside from the change from vector-valued functions to scalar-valued functions, the major difference between equation (2) and equation (5) is that in (5), the function $g(t)$ must be a function of $t$ only, and not a function of $\mathrm{x}$. That is, the white noise enters additively; it is not multiplied by any function of the solution of the differential equation.

Under this restriction, the Ito and the Stratonovich interpretations of the solution of the differential equation coincide. The divergence only arises when the white noise is multiplied by a function of the solution of the equation.

In the earlier literature, e.g. reference 1 , the stochastic differential equation (5) is called a Langevin equation. In the more mathematical modern literature, equation (5) is rewritten in a more rigorous manner. In order to avoid the mathematical pathology associated with white noise, its integral, the so-called Wiener or Brownian motion process w(t) is introduced:

$$
w(t)=\int_{0}^{t} v(t) d t
$$

The process $w(t)$ can be defined independently of $v(t)$, merely by stating that it is Gaussian and that

$$
E\{w(t)\} \equiv 0 ; E\{w(t) w(s)\}=\min \{t, s\}
$$


By "multiplying through by dt", equation (5) is recast in the form

$$
d x(t)=f(x(t), t) d t+g(t) d w(t)
$$

or by integrating once, in the form of a stochastic integral equation

$$
x(t)=x(0)+\int_{0}^{t} f(x(t), t) d t+\int_{0}^{t} g(t) d w(t)
$$

The integral on the right,

$$
\int_{0}^{t} g(t) d w(t)
$$

being an integral with respect to a Wiener process, is a new kind of integral, a so-called stochastic integral. However, so long as the function $g(t)$ is restricted as mentioned above, i.e., that it is a non-random function of $t$, then the Ito and Stratonovich interpretations of this integral agree. It may be defined, e.g., as the limit in probability of a sequence of sums of the form

$$
\sum_{i=0}^{n-1} g\left(t_{i}\right)\left[w\left(t_{i+1}\right)-w\left(t_{i}\right)\right]
$$

where $0=t_{0}<t_{1}<t_{2} \cdots<t_{n}=t$. So far there is no problem; the usual rules of calculus continue to apply to this integral.

When the engineer tells the mathematician that what he really means by a solution to the Langevin equation (5) is a solution to the integral equation (10), then the mathematician is happy, because he can prove existence and uniqueness of solutions to (10) with probability 1. Furthermore, the mathematician's solution to (10) turns out to have the sort of properties that one intuitively expects that solutions to (5) might have, so the situation is good. 
Since for each $t, x(t)$ is a random variable, it has a probability distribution associated with it. Furthermore, this distribution will be smooth enough so that it can be described by a probability density function, $p(\xi, t)$. Here the meaning of this function is that

$$
\mathrm{p}(\xi, t) \mathrm{d} \xi=\operatorname{Prob}\{\xi \leq \mathrm{x}(\mathrm{t})<\xi+\mathrm{d} \xi\}
$$

The variable $\xi$ is merely a parameter in the density function. It is not the same as the value of the process $x(t)$. In the density function $p(\xi, t)$, the two variables $\xi$ and $t$ are independent variables.

It turns out to be of great interest to study conditional densities, where we condition on the known value of the process at an earlier time. Therefore, define $p(\xi, t \mid \eta, s)$ for $s<t$ by

$$
p(\xi, t \mid \eta, s) d \xi=\operatorname{Prob}\{\xi \leq x(t)<\xi+d \xi \mid x(s)=\eta\}
$$

The function $p(\xi, t \mid \eta, s)$ will be a function of all four independent parameters $\xi, \eta, t, s$. When $t, \eta$, and $s$ are held fixed, it is a probability density function of $\xi$, e.g., $p(\xi, t \mid \eta, s) \geq 0$ and

$$
\int_{-\infty}^{\infty} p(\xi, t \mid \eta, s) d \xi=1
$$

Suppose we tried conditioning on several past events. Let $t_{1}<t_{2}<\cdots<t_{n}$. Consider the probability

$$
\operatorname{Prob}\left\{\xi \leq x\left(t_{n}\right)<\xi+d \xi \mid x\left(t_{i}\right)=\eta_{i}, \quad i=1,2, \cdots n-1\right\}
$$

It turns out that for a process $x(t)$ obtained as the solution to a stochastic integral equation of the form (10), this conditional probability is merely equal to $p\left(\xi, t_{n} \mid \eta_{n-1}, t_{n-1}\right) d \xi$. 
Written mathematically, what we are saying is.

$$
\begin{aligned}
& \operatorname{Prob}\left\{\xi \leq x\left(t_{n}\right)<\xi+d \xi \mid x\left(t_{i}\right)=\eta_{i}, \quad i=1,2, \cdots, n-1\right\} \\
& =\operatorname{Prob}\left\{\xi \leq x\left(t_{n}\right)<\xi+d \xi \mid x\left(t_{n-1}\right)=\eta_{n-1}\right\} \\
& =p\left(\xi, t_{n} \mid \eta_{n-1}, t_{n-1}\right) d \xi
\end{aligned}
$$

Any process $x(t)$ for which equation $\left(I_{4}\right)$ holds for every integer $n$, for every choice of $t_{1}, t_{2}, \cdots, t_{n}$, provided only that $t_{1}<t_{2}<\cdots<t_{n-1}$ $<t_{n}$, is called a Markov process. Stated in words, the defining property of a Markov process is that the single most recently observed value of the process contains as much information about the future evolution of the process as does knowledge of the entire past history of the process up to and including the most recently observed value.

The conditional probability density function $\mathrm{p}(\xi, t \mid \eta, s)$ plays a fundamental role in the study of continuous Markov processes. This function is customarily called the transition density for the process. The transition density $p(\xi, t \mid \eta, s)$ may be obtained by solving the forward FokkerPlanck equation (also called the forward Kolmogorov equation)

$$
\begin{gathered}
\frac{\partial p(\xi, t \mid \eta, s)}{\partial t}=-\frac{\partial}{\partial \xi}[f(\xi, t) p(\xi, t \mid \eta, s)]+\frac{I}{2} g^{2}(t) \frac{\partial^{2} p(\xi, t \mid \eta, s)}{\partial \xi^{2}} \\
-\infty<\xi<+\infty, \quad t>s
\end{gathered}
$$

with the boundary conditions

$$
\lim _{t \rightarrow s} p(\xi, t \mid \eta, s)=\delta(\xi-\eta) ; \lim _{|\xi| \rightarrow \infty} p(\xi, t \mid \eta, s)=0
$$


The transition density may equally well be obtained by solving the backward Fokker-Planck or Kolmogorov equation

$$
\begin{aligned}
& -\frac{\partial p(\xi, t \mid \eta, s)}{\partial s}=f(\eta, s) \frac{\partial p(\xi, t \mid \eta, s)}{\partial \eta}+\frac{1}{2} g^{2}(t) \frac{\partial^{2} p(\xi, t \mid \eta, s)}{\partial \eta^{2}} \\
& -\infty<\eta<+\infty, \quad s<t
\end{aligned}
$$

with the boundary conditions

$$
\lim _{s \rightarrow t} p(\xi, t \mid \eta, s)=\delta(\xi-\eta) ; \quad \lim _{|\eta| \rightarrow \infty} p(\xi, t \mid \eta, s)=0
$$

Equation (15) is a partial differential equation for $\mathrm{p}$ considered as a function of the independent variables $\xi$ and $t$. The variables $\eta$ and $s$ are merely parameters which enter through the boundary conditions (16). On the other hand, equation (17) is a partial differential equation for $p$ as a function of the independent variables $\eta$ and $s$. Here $\xi$ and $t$ are merely parameters which enter through the boundary conditions (18). The coefficient functions $f$ and $g$ are the functions defined in (5) and (6).

From an engineering standpoint, the situation may be summarized by saying that a complete probabilistic analysis of the properties of a stochastic dynamic system described by (5) may be made by finding the transition density $p(\xi, t \mid \eta, s)$ as a solution to one of the Fokker-Planck equations (if it satisfies one, it necessarily satisfies the other). This statement is accurate, provided one is careful what he does in such an analysis. The next sections will show what it means to be careful.

\section{AN APPARENT PARADOX}

Let us consider the Wiener process introduced in (7). The preceding theory applies to this process, since by setting $x(0)=0, f=0, g=1$, equation (10) becomes 


$$
x(t)=\int_{0}^{t} d w(t)
$$

i.e. $x(t)=w(t)$. In order to make our point, it will suffice to consider only the forward equation (15), and to consider its solution only for the special case of $\mathbf{s}=0, \eta=0$ in (16).

Denote this solution by $q(\xi, t)$. Thus

$$
q(\xi, t) d \xi=\operatorname{Prob}\{\xi \leq x(t)<\xi+d \xi \mid x(0)=0\}
$$

where now $\mathrm{x}(\mathrm{t})$ is a Wiener process.

It is well known that $q(\xi, t)$ is given by

$$
q(\xi, t)=\frac{1}{\sqrt{2 \pi t}} e^{-\frac{\xi^{2}}{2 t}}
$$

It is easily verified that this function obeys the forward equation

$$
\frac{\partial q(\xi, t)}{\partial t}=\frac{1}{2} \frac{\partial^{2} q(\xi, t)}{\partial \xi^{2}}
$$

and satisfies the boundary condition

$$
\lim _{t \rightarrow 0} q(\xi, t)=\delta(\xi)
$$

Now suppose the Wiener process is passed through a memoryless nonlinear device to produce a new process $z(t)$. Since the device is memoryless, the process $z(t)$ will still be Markov, and the probability density for it will obey a Fokker-Planck equation. Specifically, suppose that 


$$
z(t)=\sinh [x(t)]
$$

Define

$$
p(\zeta, t) d \zeta=\operatorname{Prob}\{\zeta \leq z(t)<\zeta+d \zeta \mid z(0)=0\}
$$

By the rule for change of variables in probability densities,

$$
p(\zeta, t)=\left.q(\xi, t) \frac{d \xi}{d \zeta}\right|_{\xi=\sinh ^{-1} \zeta}
$$

$$
\begin{aligned}
& \text { Now } \frac{d}{d \zeta} \sinh ^{-1} \zeta=\sqrt{1+\zeta^{2}} \text {, so } \\
& p(\zeta, t)=\sqrt{\frac{1+\zeta^{2}}{2 \pi t}} e^{-\frac{\left(\sinh ^{-1} \zeta\right)^{2}}{2 t}}
\end{aligned}
$$

Either by making the change of independent variable $\xi=\sinh ^{-1} \zeta$ in (22) and using (26), or by direct differentiation of (27), one finds that the FokkerPlanck equation satisfied by $p(\sigma, t)$ is

$$
\frac{\partial p(\zeta, t)}{\partial t}=-\frac{\partial}{\partial \zeta}\left[\frac{\zeta}{2} p(\zeta, t)\right]+\frac{1}{2} \frac{\partial^{2}}{\partial \zeta^{2}}\left[\left(1+\zeta^{2}\right) p(\zeta, t)\right]
$$

According to the theory in Doob (Ref. 3), this is the forward equation corresponding to the stochastic differential equation

$$
d z(t)=\frac{I}{2} z(t) d t+\left[I+z^{2}(t)\right]^{\frac{1}{2}} d w(t)
$$

This resembles the stochastic differential equation (9) discussed in the previous section. However, in regard to (9), it was specifically stated that the coefficient $g(t)$ which multiplies the noise had to be a non-random function of $t$ only. In (29), the coefficient of the noise, namely $\left[1+z^{2}(t)\right]^{\frac{1}{2}}$, is a function of $z(t)$. 
Now, if we simply compute $\mathrm{dz}(t)$ from (24) using the chain rule of ordinary calculus, we find

$$
\begin{aligned}
d z(t) & =\frac{\partial}{\partial x} \sinh x \mid \begin{array}{l}
d x(t) \\
x(t)=\sinh ^{-1}[z(t)]
\end{array} \\
& =\cosh x \mid \begin{array}{l}
d x(t) \\
x(t)=\sinh ^{-1}[z(t)]
\end{array} \\
& =\left[1+\sinh ^{2} x\right]^{\frac{1}{2}} \mid \begin{array}{l}
d x(t) \\
x=\sinh ^{-1}[z(t)]
\end{array} \\
& =\left[1+z^{2}(t)\right] d x(t)
\end{aligned}
$$

Since in the present case $x(t)=w(t)$, this may be rewritten

$$
d z(t)=\left[1+z^{2}(t)\right]^{\frac{1}{2}} d w(t)
$$

The stochastic differential equations (29) and (3I) differ by the term $\frac{1}{2} \mathrm{z} d t$. The question is, which is the correct stochastic differential equation for generating the process $z(t)$ from a Wiener process?

Ito and Doob would say that (29) is the correct equation. Stratonovich would say that (3I) is the correct equation. Let us pinpoint the exact issue of disagreement by first stating the facts on which everybody agrees.

Everybody agrees on the following:

1. The Wiener process is a well-defined process. Its probability density, given that the process starts at zero at time zero, is correctly given by (2I), and this function satisfies (22) and (23). 
2. The process $z(t)$ defined by $(24)$ is a well-defined process. Its density function, defined in (25), is correctly given by (27), and this function satisfies (28). Thus, in particular, both Stratonovich and Ito would agree that (28) is the correct Fokker-Planck equation for the $\mathrm{z}(\mathrm{t})$ process defined by $(24)$.

3. Everybody agrees that if we integrate (3I) according to the mules of ordinary calculus we do get $z(t)=\sinh [w(t)]$ as the solution, while if we integrate (31) according to the Ito calculus we do not get this as the solution.

4. Ito and Stratonovich would both agree that if we integrate (29) according to the rules of Ito calculus we do get $z(t)=\sinh [w(t)]$ as the solution, while if we integrate (3I) according to ordinary calculus, we do not get this as a solution.

Therefore, the situation is that the one unambiguous way to specify a Markov process mathematically is to specify its transition density, or equivalently, the Fokker-Planck equation obeyed by the transition density. The divergence arises when one wishes to generate the specified process as a solution to a stochastic differential equation forced by the differential of a Wiener process. The divergence boils down to two different ways of associating the coefficients in the Fokker-Planck equation with the coefficients in the stochastic differential equation, and respectively two ways of integrating this stochastic equation.

Each way is consistent within itself, as we have seen. Starting from the process $z(t)$ defined by the Fokker-Planck equation (28), the use of Stratonovich rules associates the stochastic differential equation (3I) with (28). Integrating (3I) by the Stratonovich rules yields $z(t)=\sinh [w(t)]$.

On the other hand, the use of Ito rules will associate the stochastic differential equation (29) with the Fokker-Planck equation (28). However, integrating (29) by the Ito rules again yields $z(t)=\sinh [w(t)]$. Further, 
Ito would say that the computation of the differential $\mathrm{dz}(\mathrm{t})$ in (30) is incorrect; if this computation is done by Ito rules then (29) results. However, Stratonovich would say that (30) is a perfectly valid computation.

At first glance, it might seem academic to worry about this divergence between Ito rules and Stratonovich rules. Each set of mules is consistent within itself. If the same set of rules is consistently applied throughout the whole computation, both methods yield the same result.

The mathematician discusses Markov process by starting with the transition density for the process. He is able to associate a FokkerPlanck equation in an unambiguous way with this transition density. When he finds that he has two possible ways of modelling the process as the solution to a stochastic differential equation, he will choose the way which has the most mathematical elegance in its internal structure, and which is capable of the greatest generalization. Considered from this standpoint, the Ito calculus is the "right" choice. Indeed, the procedure just described is precisely the one followed by Doob in his book (reference 3 ).

However, the question is not so simple for the engineer. He cannot resolve the issue on the basis of mathematical elegance alone. The engineer does not start with the transition density. As discussed in the earlier sections, the engineer starts with a differential equation which he has obtained on the basis of known physical laws. He then adds a white noise forcing term to get a stochastic model. If the coefficient of the noise is itself random, then there are two possible ways of interpreting the equation, leading to two different Fokker-Planck equations and two different processes. The question is, which process does one "really" get in the physical world? Which kind of calculus does nature use?

The answer to this question hinges on whether white noise "really" exists, or whether the concept of white noise is only a convenient approximation which we use in place of a more detailed knowledge of the properties of the noise process. The true situation is certainly the latter, since noise with a truly flat power density spectrum out to infinite frequency would carry infinite total power. However, this 
then implies that there is really no such thing as a Markov process either, and the whole theory of the Fokker-Planck equation goes down the drain.

Therefore, the whole theory of white noise, stochastic differential equations, Markov processes, and the Fokker-Planck equation must be approached from the standpoint of an approximate model rather than an exact model of physical reality. It is, of course, possible to use non-white noise in the model, but now one is faced with the problem of specifying the power density spectrum of the noise, which is usually completely unknown at high frequencies, even though it can be measured as flat at low frequencies. Furthermore, use of a non-flat high frequency spectrum complicates the computations tremendously.

Once one realizes the kind of approximation that is being made, it turns out that it is possible to use either the Ito or the Stratonovich mules and obtain equally accurate results, provided that one is careful in setting up the mathematical model and that one is aware of the subtleties involved.

The paradox of obtaining two different stochastic processes as solutions to the same stochastic differential equation thus turns out to arise from the pathological nature of white noise. This paradox can be avoided by treating this pathology with proper respect. In the following sections we will examine the situation in more detail.

\section{THE ITO CALCULUS}

In order to introduce the Ito calculus, let us begin by examining the wiener process $w(t)$ more carefully. Let $\Delta t$ be some very small, but not infinitesimal, increment of time. Define

$$
\Delta w(t)=w(t+\Delta t)-w(t)
$$


For fixed $t$ and $\Delta t, w(t+\Delta t)$ and $w(t)$ are both Gaussian random variables, so $\Delta w(t)$ is also a Gaussian random variable.

Let $q(\xi, t \mid \eta, s)$ be the transition density for the Wiener process, ie.

$$
\mathrm{q}(\xi, \mathrm{t} \mid \eta, \mathrm{s}) \mathrm{d} \xi=\operatorname{Prob}\{\xi \leq \mathrm{w}(\mathrm{t})<\xi+\mathrm{d} \xi \mid \mathrm{w}(\mathrm{s})=\eta\}
$$

By the definition of the Wiener process, this density is given by

$$
q(\xi, t \mid \eta, s)=\frac{1}{\sqrt{2 \pi(t-s)}} e^{-\frac{(\xi-\eta)^{2}}{2(t-s)}}
$$

With somewhat of an abuse of notation, define the conditional probability density

$$
\mathrm{p}_{\Delta \mathrm{w}}(\Delta \xi \mid \xi) \mathrm{d}(\Delta \xi)=\operatorname{Prob}\{\Delta \xi \leq \Delta \mathrm{w}(\mathrm{t})<\Delta \xi+\mathrm{d}(\Delta \xi) \mid \mathrm{w}(\mathrm{t})=\overline{\mathrm{s}}\}
$$

Since we are conditioning on the fixed event $\{w(t)=\xi\}$, observe that

$$
\begin{aligned}
\operatorname{Prob}\{\Delta \xi & \leq \Delta \mathrm{w}(\mathrm{t})<\Delta \xi+\mathrm{d}(\Delta \xi) \mid \mathrm{w}(\mathrm{t})=\xi\} \\
& =\operatorname{Prob}\{\xi+\Delta \xi \leq \mathrm{w}(\mathrm{t})+\Delta \mathrm{w}(\mathrm{t})<\xi+\Delta \xi+\mathrm{d}(\Delta \xi) \mid \mathrm{w}(\mathrm{t})=\xi\} \\
& =\operatorname{Prob}\{\xi+\Delta \xi \leq \mathrm{w}(\mathrm{t}+\Delta \mathrm{t})<\xi+\Delta \xi+\mathrm{d}(\Delta \xi) \mid \mathrm{w}(\mathrm{t})=\xi\}
\end{aligned}
$$

It follows from (33) - (36) that

$$
\begin{aligned}
\mathrm{p}_{\Delta \mathrm{w}}(\Delta \xi \mid \xi) & =\mathrm{q}(\xi+\Delta \xi, \mathrm{t}+\Delta \mathrm{t} \mid \xi, \mathrm{t}) \\
& =\frac{1}{\sqrt{2 \pi[(t+\Delta t)-t]}} \exp \left\{\frac{[(\xi+\Delta \xi)-\xi]^{2}}{2[(t+\Delta t)-t]}\right\}
\end{aligned}
$$

21 


$$
=\frac{1}{\sqrt{2 \pi \Delta t}} e^{-\frac{(\Delta \xi)^{2}}{2 \Delta t}}=p_{\Delta w}(\Delta \xi)
$$

In the last line of (37) we have written $\mathrm{p}_{\Delta \mathrm{W}}(\Delta \xi)$ to denote the unconditional probability density for the random variable $\Delta w(t)$, i.e.

$$
\mathrm{P}_{\Delta \mathrm{W}}(\Delta \xi) \mathrm{d}(\Delta \xi)=\operatorname{Prob}\{\Delta \xi \leq \Delta \mathrm{W}(\mathrm{t})<\Delta \xi+\mathrm{d}(\Delta \xi)\}
$$

The important point is that

$$
\mathrm{P}_{\Delta \mathrm{W}}(\Delta \xi \mid \xi)=\mathrm{P}_{\Delta \mathrm{W}}(\Delta \xi)
$$

1.e., the distribution of the increment $\Delta_{w}(t)$ is independent of $w(t)$, the state of the process at time $t$. This is not generally true of random processes, or even of Markov processes. The Wiener process $w(t)$ belongs to a special class of processes known as processes with independent increments.

From (37) and (38), we see that the random variable $\Delta W(t)$ defined in (32) is gaussian with mean zero and variance $\Delta t$. The fact that $E\left\{(\Delta \mathrm{W})^{2}\right\}$ is first order in $\Delta t$ is what causes the peculiarities of the Ito stochastic calculus.

Let $\mathrm{F}$ be any smooth real-valued nonlinear function of a real variable. Consider $F(W(t+\Delta t))$, where $W(t)$ continues to denote the wiener process. By Taylor series and (32),

$$
\begin{gathered}
F(w(t+\Delta t))=F(w(t)+\Delta w(t)) \\
=F(w(t))+F^{\prime}(w(t)) \Delta w(t)+\frac{1}{2} F^{\prime \prime}(w(t))[\Delta w(t)]^{2}+\cdots
\end{gathered}
$$

Using the distribution of $\Delta w(t)$, we have

$$
\begin{gathered}
E\left\{(\Delta \mathrm{w})^{\mathrm{k}}\right\}=0, \mathrm{k} \text { odd } \\
\mathrm{E}\left\{(\Delta \mathrm{w})^{\mathrm{k}}\right\}=1.3 .5 \ldots(\mathrm{k}-1)(\Delta \mathrm{t})^{\mathrm{k} / 2}, \mathrm{k} \text { even }
\end{gathered}
$$


Use the notation $0(\Delta t)$ to denote a remainder consisting only of terms of order $(\Delta t)^{2}$ and higher.

Suppose now we tried to define

$$
\frac{d}{d t} F(w(t))=\lim _{\Delta t \rightarrow 0} \frac{F(w(t+\Delta t))-F(w(t))}{\Delta t}
$$

From (40) and (41), it follows that

$$
\begin{aligned}
E\left\{\frac{\mathrm{d}}{\mathrm{dt}} F(w(t))\right\} & =\lim _{\Delta t \rightarrow 0} \frac{\frac{1}{2} E\left\{F^{\prime \prime}(w(t))\right\} \Delta t+0(\Delta t)}{\Delta t} \\
& =\frac{1}{2} E \quad\left\{F^{\prime \prime}(w(t))\right\}
\end{aligned}
$$

On the other hand, if one computer the total differential $d F(w(t))$ using the chain rule of ordinary calculus, one has

$$
\mathrm{dF}(\mathrm{W}(t))=\mathrm{F}^{\prime}(\mathrm{w}(t)) \mathrm{dw}(t)
$$

By passing from an increment $\Delta w(t)$ to a differential dw(t), it follows from (39) that $d w(t)$ is independent of $w(t)$. Therefore,

$$
\begin{gathered}
E\{d F(w(t))\}=E\left\{F^{\prime}(w(t)) d w(t)\right\} \\
=E\left\{F^{\prime}(w(t))\right\} E\{d w(t)\}=0
\end{gathered}
$$

since $E\{d w(t)\}=0$ by (41). Now (45) would imply that

$$
E\left\{\frac{d}{d t} F(w(t))\right\}=0
$$

in contradiction to (43).

The point is that because of the fact that $E\left\{(\Delta w)^{2}\right\}=\Delta t$, the definition of the derivative (42) no longer leads to the usual rules of calculus. Ito was the first to show how the rules of calculus should be modified to handle this phenomenon. First of all, instead of computing the derivative as in (42), one should compute the differential dF(t), because the differential $d_{W}(t)$ can be rigorously interpreted whereas the derivative $\frac{d w(t)}{d t}$ can not. 
As given in reference (4), the Ito rule for the stochastic differential in the present case is

$$
d_{I} F(w(t))=F^{\prime}(w(t)) d w(t)+\frac{1}{2} F^{\prime}(w(t)) d t
$$

where now $d_{I}$ means Ito differential. Note that this rule is now consistent with Doob's treatment of the Fokker-Planck equation. Let us apply (47) in the special case when $F(w(t))=\sinh [w(t)]$.

$$
\begin{aligned}
& \text { Now } F^{\prime}(W)=\cosh W, F^{\prime \prime}(W)=\sinh w \text {, so }(47) \text { says } \\
& \qquad d_{I} \sinh [W(t)]=\cosh w(t) d w(t)+\frac{1}{2} \sinh w(t) d t
\end{aligned}
$$

Let us write $z=\sinh w$. Then, $\cosh w=\left[1+z^{2}\right]^{1 / 2}$, so (48) can be rewritten as

$$
a_{I} z(t)=\frac{1}{2} z(t) d t+\left[1+z^{2}(t)\right]^{1 / 2} d w(t)
$$

which is the same as (29). Thus, the Ito rule (47) for the total differential is consistent with the Fokker-Planck equation (28).

Since the rule for computing total differentials has now been changed from (44) to (47), we must expect a corresponding change in the rule for integration. Let us write (I) $\int$ when an integral is to be understood in the Ito sense, and continue to write just $f$ for ordinary integrals.

We wish to preserve the fundamental property of calculus, that the integral can be interpreted as an anti-derivative. Therefore, we require that

$$
\text { (I) } \int_{t_{0}}^{t_{1}} d_{I} F(w(t))=F\left(w\left(t_{1}\right)\right)-F\left(w\left(t_{0}\right)\right)
$$

Applying this to (47) yields

$$
\begin{array}{r}
\text { (I) } \int_{t_{0}}^{t_{1}} d_{I} F(w(t))=F\left(w\left(t_{1}\right)\right)-F\left(w\left(t_{0}\right)\right) \\
=(I) \int_{t_{0}}^{t_{1}^{1}} F^{\prime}(w(t)) d w(t)+\frac{1}{2} \int_{t_{0}}^{t_{1}} F^{\prime}(w(t)) d t
\end{array}
$$


This may be rewritten as

(I) $\int_{t_{0}}^{t_{I}} F^{\prime}(w(t)) d w(t)=F\left(w\left(t_{1}\right)\right)-F\left(w^{\prime}\left(t_{0}\right)\right)-\frac{1}{2} \int_{t_{0}}^{t_{I}} F^{\prime \prime}(w(t)) d t$

Now let $g(x)$ be any once-differentiable function. Define

$$
G(x)=\int_{0}^{x} g(\xi) d \xi
$$

Then, using (52) with $F$ replaced by G,

(I) $\int_{t_{0}}^{t_{I}} g(w(t)) d w(t)=(I) \int_{t_{0}}^{t_{1}} G^{\prime}(w(t)) d w(t)$

$$
\begin{aligned}
& =G(w(t))-G\left(w\left(t_{0}\right)\right)-\frac{1}{2} \int_{t_{0}}^{t_{1}} G^{\prime \prime}(w(t)) d t \\
& =\int_{w\left(t_{0}\right)}^{w(t)} g(\xi) d \xi-\frac{1}{2} \int_{t_{0}}^{t_{1}} g^{\prime}(w(t)) d t
\end{aligned}
$$

In $(54)$, the notation $\int_{W\left(t_{0}\right)}^{W\left(t_{1}\right)} g(\xi) d \xi$ means compute $\int g(\xi) d \xi$ as

an ordinary integral, treating $\xi$ as a deterministic dummy variable of integration, and then evaluate between the random limits $w\left(t_{l}\right)$ and $w\left(t_{0}\right)$. This, incidentally, is essentially what stratonovich has in mind in his definition of the stochastic integral. 
Let (s) $\int$ denote the Stratonovich integral. Then, in the present context,

(S) $\int_{t_{0}}^{t_{1}} g(w(t)) d w(t)=\int_{w\left(t_{0}\right)}^{w\left(t_{1}\right)} g(\xi) d \xi$

Therefore, (54) can be rewritten

(I) $\int_{t_{0}}^{t_{I}} g(w(t)) d w(t)=$ (S) $\int_{t_{0}}^{t_{I}} g(w(t)) d w(t)-\frac{1}{2} \int_{t_{0}}^{t_{I}} g^{\prime}(w(t)) d t(56)$

which is a special case of the formula given by Stratonovich in reference (5) for the connection between Ito integrals and Stratonovich integrals.

The Ito calculus has some surprising consequences. For example, let $g(w(t))=w(t)$ in $(56)$. By the notation $g^{\prime}(w(t))$ we mean, of course,

$$
g^{\prime}(w(t))=\left.\frac{d g(\xi)}{d \xi}\right|_{\xi=w(t)}
$$

so that in the present case $g^{\prime}(w(t))=1$. Now,

$$
\int \xi \mathrm{d} \xi=\frac{1}{2} \xi^{2}
$$

so using (55) and (56) we obtain

(I) $\int_{t_{0}}^{t_{1}} w(t) d w(t)=\frac{1}{2} w^{2}\left(t_{1}\right)-\frac{1}{2} w^{2}\left(t_{0}\right)-\frac{1}{2}\left(t_{1}-t_{0}\right)$

an example which is also given by Doob. 
The presence of the $\frac{1}{2}\left(t_{1}-t_{0}\right)$ term in (59) can be made more plausible by the following considerations. Let us consider

$$
E\left\{(I) \int_{t_{0}}^{t_{1}} w(t) d w(t)\right\}=\int_{t_{0}}^{t_{1}} E\{w(t) d w(t)\}
$$

As in (45), we have

$$
E\{w(t) d w(t)\}=E\{w(t)\} E\{d w(t)\}=0
$$

since the increment $d w(t)$ is understood to be independent of $w(t)$. Therefore, we conclude

$$
E\left\{(I) \int_{t_{\sigma}}^{t_{I}} w(t) d w(t)\right\}=0
$$

Now recall that the wiener process was defined such that $w(0)=0$. Thus, by (21) we have that

$$
E\left\{w^{2}\left(t_{1}\right)\right\}=t_{1} ; \quad E\left\{w^{2}\left(t_{0}\right)\right\}=t_{0}
$$

Taking the expected value of both sides of (59) now gives

$$
\begin{aligned}
E\left\{(I) \int_{t_{0}}^{t_{I}} w(t) d w(t)\right\} & =\frac{1}{2} E\left\{w^{2}\left(t_{1}\right)\right\}-\frac{1}{2} E\left\{w^{2}\left(t_{0}\right)\right\}-\frac{1}{2}\left(t_{1}-t_{0}\right) \\
& =\frac{1}{2} t_{1}-\frac{1}{2} t_{0}-\frac{1}{2}\left(t_{1}-t_{0}\right)=0
\end{aligned}
$$

in agreement with (62). Thus, the $\frac{1}{2}\left(t_{1}-t_{0}\right)$ can be viewed as a correction term which insures that (62) holds. 
However, these considerations also imply that for the Stratonovich integral,

$$
E\left\{(S) \int_{t_{0}}^{t_{1}} w(t) d w(t)\right\}=\frac{1}{2}\left(t_{1}-t_{0}\right) \neq 0
$$

Thus, for the Stratonovich integral, it cannot be true that $\mathrm{dw}(t)$ is independent of $w(t)$, for we have just seen that this independence is what makes the expected value of the Ito integral always zero.

In fact, this is precisely the case. Stratonovich interprets the differential $d w(t)$ in such a way that it is not independent of $w(t)$.

The Ito calculus is based on the fact that the increment $\Delta w(t)$ defined in (32) is independent of $\mathrm{w}(\mathrm{t})$, and has mean zero and variance $\Delta t$.

In contract, Stratonovich works with a "Stratonovich increment" defined as

$$
\Delta_{s} w(t)=w\left(t+\frac{\Delta t}{2}\right)-w\left(t-\frac{\Delta t}{2}\right)
$$

This increment still has mean zero and variance $\Delta t$, but it is not independent of $w(t)$. We will examine the Stratonovich calculus in more detail in the next section.

This report is written in such a way as to be (we hope) pedagogically palatable to engineers. Consequently, our treatment of stochastic differential equations and stochastic calculus differs drastically from the rigorous mathematical treatment given in references 3 and 4 . Rather than carefully stating and proving theorems, we are trying to convey the basic ideas involved by considering only special cases and examining illustrative examples.

So far, we have discussed the Ito calculus by following the approach historically used in presenting ordinary calculus to students for the first 
time. Namely, we introduced the derivative first, as the sort of limit given in (42). The integral was then introduced as an antiderivative.

In the modern, rigorous approach to calculus, which is usually presented to students only after their intuition has been sharpened, the integral is defined directly from first principles. The Riemann integral is defined as a limit of Riemann sums, and the Lebesgue integral is defined by use of measure theory.

Similarly, in a rigorous approach to stochastic calculus, the Ito integral is defined first, as a stochastic limit of Riemann-type sums. The Ito differential formula (47) is then derived as a consequence of this integral.

Let us sketch briefly the definition of the Ito integral as a limit of sums. Let $w(t)$ be a Wiener process. Let $z(t)$ be any random process having the properties that, for all $t, z(t)$ and $[w(\tau)-w(t)]$ are independent for all $\tau>t$, and that

$$
\int_{0}^{T} z^{2}(t) d t<\infty
$$

with probability one. Note that, for $\tau \leq t, z(t)$ and $[w(\tau)-w(t)]$ may be dependent. Let $0=t_{0}<t_{1}<t_{2}<\cdots<t_{n}=T$. Let

$$
\Delta_{n}=\max _{1 \leq i \leq n}\left|t_{i}-t_{i-1}\right|
$$

Choose any sequence of partitions $t_{0}, t_{1}, \cdots, t_{n}$ such that

$\lim _{n \rightarrow \infty} \Delta_{n}=0$ 
The object is to define the Ito integral

$$
J=(I) \int_{0}^{T} z(t) d w(t)
$$

Define

$$
I_{n}=\sum_{k=1}^{n} z\left(t_{k-1}\right)\left[w\left(t_{k}\right)-w\left(t_{k-1}\right)\right]
$$

Note that the integrand $z\left(t_{k-1}\right)$ is always evaluated at the beginning of the interval $\left[t_{k-1}, t_{k}\right]$ over which the increment $\left[w\left(t_{k}\right)-w\left(t_{k-1}\right)\right]$ is taken. Therefore, $z\left(t_{k-1}\right)$ and $\left[w\left(t_{k}\right)-w\left(t_{k-1}\right)\right]$ are always independent. Consequently,

$$
E\left\{I_{n}\right\}=\sum_{k=1}^{n} E\left\{z\left(t_{k-I}\right)\right\} E\left\{w\left(t_{k}\right)-w\left(t_{k-I}\right)\right\}=0
$$

It is now possible to prove that the sequence of random variables $I_{n}$ converges in probability to some limiting random variable $\mathrm{J}$. This limit is called the Ito integral. It has the property that $E\{J\}=0$.

Note that the class of random processes $z(t)$ which may be used as integrands here is very broad. It is only required that $z(t)$ be square integrable over the interval of integration and that the present value of $z(t)$ is always independent of all future increments of $w(t)$. In fact, there is not even any reason why the integrating process $w(t)$ has to be a Wiener process. References 3 and 4 discuss this in detail. The point is that the definition of the stochastic integral given by Ito is really quite general, much more so than our heuristic derivation of (56) would indicate. 
VII. THE STRATONOVICH CALCULUS

In the previous section we asserted that a derivative defined as a limit of the form $(42)$ is consistent with an integral defined as a limit of sums of the form (70), and we gave some examples to make this assertion plausible. The resulting stochastic calculus is called the Ito calculus. By examples such as (48) and (59) it was illustrated that the rules of the Ito calculus differ from the usual rules of ordinary calculus.

In reference (5), Stratonovich proposed a definition of the stochastic integral under rather restrictive conditions which leads to a stochastic calculus whose rules are the same as ordinary calculus. Basically, what Stratonovich did was to show that the formula (56) could be made rigorous. Thus, with the Ito integral on the left-hand side of (56) already welldefined, the Stratonovich integral on the right-hand side of (56) becomes well-defined.

Therefore, Stratonovich did not give a fundamental definition of a new stochastic integral, but only defined the new integral in terms of the already existing Ito integral. Furthermore, the new integral is not defined for forms as general as (69). It is only defined for the special case of (69) in which $z(t)$ is of the form

$$
z(t)=g(w(t), t)
$$

where $g(x, t)$ is a non-random function of the two arguments $x$, $t$. Consequently, the Ito integral remains both more fundamental and more general than the Stratonovich integral.

It is tempting to suppose that a fundamental definition of the Stratonovich integral could be given, in anology with (70), by taking a sequence of sums of the forin 


$$
I_{n}^{*}=\sum_{k=1}^{n} z\left(\frac{t_{k-1}+t_{k}}{2}\right)\left[w\left(t_{k}\right)-w\left(t_{k-1}\right)\right]
$$

Unfortunately, such a sequence of sums cannot be shown to converge, in general, even in such a weak sense as convergence in probability. The Stratonovich integral is not versatile enough to be suited for many applications for which the Ito integral is suited.

The Stratonovich integral is just versatile enough to be suited to the integration of stochastic differential equations. Consider the following generalization of (9).

$$
d x(t)=f(x(t), t) d t+g(x(t), t) d w(t)
$$

The functions $f(x, t)$ and $g(x, t)$ are assumed to be jointly continuous in $x$ and $t$, once differentiable with respect to $x$, and to satisfy the following condition: there exist constants $K_{1}, K_{2}<\infty$ such that

$$
\begin{aligned}
& |f(x, t)| \leq K_{1}+K_{2}|x| \\
& |g(x, t)| \leq K_{1}+K_{2}|x|
\end{aligned}
$$

for all $t$ and all $x$.

By rewriting (74) as an integraI equation, one obtains

$$
x(t)=x(0)+\int_{0}^{t} f(x(\tau), \tau) d \tau+\int_{0}^{t} g(x(\tau), \tau) d w(\tau)
$$


The stochastic integral on the right has as its integrand $g(x(\tau), \tau)$, rather than $g(w(\tau), \tau)$ as is required by (72). However, by giving a multi-dimensional definition of his integral, Stratonovich was able to show how the integral in (75) could be recast in the desired form. Therefore, it is possible to say that the stochastic integral on the righthand side of (75) can be interpreted as a Stratonovich integral. That is, Stratonovich integrals of the form

$$
\int_{0}^{T} g(x(t), t) d w(t)
$$

can be defined, provided $d x(t)$ and $d w(t)$ are connected by a stochastic differential equation such as (74). This is apparently the most general situation for which the Stratonovich integral can be defined.

It is now possible to give an existence and uniqueness proof of solutions to the stochastic integral equation (75) when the stochastic integral is interpreted in the Stratonovich sense, in analogy to the type of proof using Picard iteration that Doob gives for the case of an Ito integral.

The Stratonovich and the Ito solutions of (75) will of course be different, because of the divergence between the two integrals indicated by (56). Call $x_{I}(t)$ the Ito solution and $x_{S}(t)$ the Stratonovich solution. Explicitly, we have

$$
\begin{aligned}
& x_{I}(t)=x_{I}(0)+\int_{0}^{t} f\left(x_{I}(\tau), \tau\right) d \tau+(I) \int_{0}^{t} g\left(x_{I}(\tau), \tau\right) d w(\tau) \\
& x_{s}(t)=x_{s}(0)+\int_{0}^{t} f\left(x_{s}(\tau), \tau\right) d \tau+(s) \int_{0}^{t} g\left(x_{s}(\tau), \tau\right) d w(\tau)
\end{aligned}
$$


Although $x_{I}(t)$ and $x_{s}(t)$ are two different processes, they both still turn out to be Markov processes. Call $\rho_{I}(\xi, t / \eta, s)$ the transition density associated with $x_{I}(t)$, and $\rho_{s}(\xi, t \mid \eta, s)$ the transition density associated with $x_{s}(t)$.

As given by Doob, $\rho_{I}(\xi, t \mid \eta, s)$ obeys respectively the forward and backward Kolmogorov equations

$$
\begin{aligned}
& \frac{\partial \rho_{I}(\xi, t \mid \eta, s)}{\partial t}=-\frac{\partial}{\partial \xi}\left[f(\xi, t) \rho_{I}(\xi, t \mid \eta, s)\right]+\frac{1}{2} \frac{\partial^{2}}{\partial \xi^{2}}\left[g(\xi, t) \rho_{I}(\xi, t \mid \eta, s)\right] \\
& -\frac{\partial \rho_{I}(\xi, t \mid \eta, s)}{\partial s}=f(\eta, s) \frac{\partial \rho_{I}(\xi, t \mid \eta, s)}{\partial \eta}+\frac{1}{2} g^{2}(\eta, s) \frac{\partial^{2} \rho_{I}(\xi, t \mid \eta, s)}{\partial \eta^{2}}
\end{aligned}
$$

On the other hand, Stratonovich shows that $\rho_{S}(\xi, t \mid \eta, s)$ obeys respectively the forward and backward equations

$$
\begin{aligned}
& \frac{\partial \rho_{s}(\xi, t \mid \eta, s)}{\partial t}=-\frac{\partial}{\partial \xi}\left[f(\xi, t) \rho_{s}(\xi, t \mid \eta, s)\right]+\frac{1}{2}\left\{\frac{\partial}{\partial \xi} g(\xi, t) \frac{\partial}{\partial \xi}\left[g(\xi, t) \rho_{s}(\xi, t \mid \eta, s)\right]\right\} \\
& (80), \\
& \frac{\partial \rho_{s}(\xi, t \mid \eta, s)}{\partial s}=f(\eta, s) \frac{\partial \rho_{s}(\xi, t \mid \eta, s)}{\partial \eta}+\frac{1}{2} g(\eta, s) \frac{\partial}{\partial \eta}\left[g(\eta, s) \frac{\partial \rho_{s}(\xi, t \mid \eta, s)}{\partial \eta}\right]
\end{aligned}
$$

We saw earlier that if (29) is interpreted in the Ito sense, then the appropriate forward Kolmogorov equation is (28). If one now uses the Stratonovich rule (80) for the forward equation, one finds that if (31) is interpreted in the Stratonovich sense, then the appropriate forward equation is again (28). This is as it should be, since the Ito solution of (29) and the Stratonovich solution of (3I) are the same process, namely 


$$
z(t)=\sinh [w(t)]
$$

as we saw earlier.

This would suggest that it ought to be possible to obtain the Ito solution $x_{I}(t)$ of (76) also as the solution of some Stratonovich equation, and vice versa. Indeed, this turns out to be the case. It was shown in both references $(5)$ and $(8)$ that $x_{I}(t)$ also obeys

$$
\begin{aligned}
x_{I}(t)= & x_{I}(0)+\int_{0}^{t}\left[f\left(x_{I}(\tau), \tau\right)-\frac{1}{2} g\left(x_{I}(\tau), \tau\right) g_{I}\left(x_{I}(\tau), \tau\right)\right] d \tau \\
& +(S) \int_{0}^{t} g\left(x_{I}(\tau), \tau\right) d w(\tau)
\end{aligned}
$$

where

$$
g_{1}(x, t)=\frac{\partial g(x, t)}{\partial x}
$$

Similarly, the solution $x_{s}(t)$ of $(77)$ also obeys

$$
\begin{aligned}
x_{s}(t)= & x_{s}(0)+\int_{0}^{t}\left[f\left(x_{s}(\tau), \tau\right)+\frac{1}{2} g\left(x_{s}(\tau), \tau\right) g_{I}\left(x_{s}(\tau), \tau\right)\right] d \tau \\
& +(I) \int_{0}^{t} g\left(x_{s}(\tau), \tau\right) d w(\tau)
\end{aligned}
$$

Therefore, although the Ito integral is more fundamental and more general than the Stratonovich integral, it turns out that when we restrict 
our attention to Stochastic differential equations of the form (74), the two definitions of the stochastic integral lead to two different, but interchangeable, theories.

\section{VIII。 MODELING THE REAL WORLD}

We saw in the last section that the stochastic differential equation (74) is ambiguous. The ambiguity may be removed by writing the equation in integral form with the type of integral definitely indicated, as in (76) and (77).

We now return to the situation discussed at the beginning of this report. Suppose an engineer has a deterministic model of a dynamic system of the form of equation (I). Suppose that he wants now to include the effects of stochastic forces in the environment, and that physical reasoning suggests that a plausible stochastic model is equation (2). Which way should he interpret this equation, Ito or Stratonovich? Which kind of stochastic integration does Nature herself perform?

In order to answer this question, it must be kept clearly in mind exactly what is the purpose of a mathematical model. Presumably we have in front of us a physical dynamic system, i.e. a "black box", whose output is a random process. For simplicity, suppose this random process is scalar-valued, and call it $y(t)$.

In order to take advantage of the theory of Markov processes, one wishes to obtain $y(t)$ by means of a state-output relation of the form

$$
y(t)=h(x(t), t)
$$

where $x(t)$ is an $n$-dimensional vector-valued Markov process. The value of $n$, the statistics of the process $x(t)$, and the deterministic function 
$h$ are to be chosen in some suitable way so that the statistical properties of the process $y(t)$ obtained from (86) approximate to an acceptable degree of accuracy the sample statistics of the observed output of the black box.

It will further be convenient to obtain the Markov process $x(t)$ by means of a stochastic differential equation of the form (2). Once the statistics of the $x(t)$ process have been specified, we have seen in the previous sections how the functions $f$ and $G$ may be chosen so that either the Ito or the Stratonovich interpretation may be used.

Since the form of the function $h$ in (86) and the coordinatization of the state space are at our disposal, one may be able to make this choice in such a way that the matrix $G$ in (2) is not a function of $x(t)$, i.e. $G$ would be a purely deterministic function of time. In this case, it is possible to avoid the Ito-Stratonovich divergence altogether, as we have seen.

The point of view being taken here is that the modeling problem consists of trying to make the statistics of the output of the mathematical model agree with the statistics of the physically observable output of a given black box. There is no claim that equations (2) and (86) "really" portray what is "actually happening" inside the box, since the inside of the box is not observable to us.

This philosophical approach to the problem is generally known as the phenomenological approach, in contrast to what might be called an axiomatic approach.

If one adopts this phenomenological approach of working backwards from the output with the only objective being to match the generated output with the observed data, then the choice between the Ito and Stratonovich calculi becomes merely a matter of personal preference. On this level, mathematicians will prefer the Ito calculus because of its elegance and generality, while engineers will prefer the stratonovich calculus because of their familiarity with its rules. 
It seems to the present author that this is perhaps the best resolution of the controversy, since it avoids having to answer the question of whether "nature" prefers Ito integrals or Stratonovich integrals.

Another way of reaching essentially the same conclusion is to realize that true white noise cannot exist in the physical world. Any noise process, regardless of how flat its power density spectrum appears at low frequencies, must have a spectrum which eventually drops off to zero at sufficiently high frequencies, in order for the total power carried by the process to be finite. Physically, the dropping off of the spectrum may occur because of quantum mechanical effects if for no other reason. White noise is reminiscent of the "ultraviolet catastrophe" which appeared when black-body radiation was treated by classical physics.

Consequently, as pointed out previously, the concepts of the Wiener process and of a Markov process are mathematical idealizations which can only approximate physical reality.

Suppose we have a sequence of continuous-time stochastic processes, of finite total power, which become better and better approximations of white noise as one passes to the limit. In references (7) and (8), the point is made that the Ito and Stratonovich integrals behave differently under passage to the limit. Our point here is that this is no cause for concern, provided that one understands what is happening and views it appropriately, because Nature herself never passes to the limit.

For example, if one wishes to simulate equation (2) on a digital computer, since the digital computer operates necessarily in discrete time, the simulation output will be a discrete-time approximation to the desired continuous-time process. It is known how to program the computer so that its output will approximate either the Ito solution of (2) or the Stratonovich solution of (2) to any reasonable accuracy. 
The same remarks apply to analog simulation. Now, the analog computer operates in continuous time, but since it must necessarily employ a physical noise generator, the spectrum of the noise cannot be truly white. This is in contrast to the digital computer, where it is possible to obtain true discrete-time white noise. Nevertheless, Professor T. Kailath of Stanford mentioned in a recent talk (reference 9) a way of rigging the analog computer so that it will approximate either Ito integration or Stratonovich integration.

The above remarks still have not answered the question of what an engineer should do when he already has a deterministic model of a physical system, and he wants to convert it to a stochastic model. The safest answer is that he should throw away the deterministic model, and remodel the whole problem, with the objective being to get the statistics of the output of a Monte Carlo computer simulation to agree with the statistics of the observed data from the physical system. Any effort less than this is an attempt to find a shortcut, and may yield an incorrect model.

As an example of the kind of situation that may occur in modeling, consider the planar motion of a particle of unit mass, subject to no deterministic forces.

In inertially fixed Cartesian coordinates, the dynamic equations of motion (analogous to equation (I)) are

$$
\begin{aligned}
& \dot{v}_{x}(t)=0 \\
& \dot{v}_{y}(t)=0
\end{aligned}
$$

Suppose we introduce flight path coordinates and write $v_{X}=V \cos \beta$, $\mathrm{v}_{\mathrm{y}}=\mathrm{V} \sin \beta$. The flight path equations of motion are

$$
\begin{aligned}
\dot{V} & =0 \\
V \dot{\beta} & =0
\end{aligned}
$$


If one integrates both (87) and (88), starting from corresponding initial conditions, both (87) and (88) yield the same straight line for a trajectory.

Now consider making the jump from equation ( 1 ) to equation (2). Let $n_{x}(t)$ and $n_{y}(t)$ be independent Gaussian white noises, each of unity power density. Equation (87) becomes

$$
\begin{aligned}
& \dot{v}_{x}(t)=n_{x}(t) \\
& \dot{v}_{y}(t)=n_{y}(t)
\end{aligned}
$$

In order to write these equations in Ito form, introduce the two Wiener processes

$$
\begin{aligned}
& w_{x}(t)=\int_{0}^{t} n_{x}(\tau) d \tau \\
& w_{y}(t)=\int_{0}^{t} n_{y}(\tau) d \tau
\end{aligned}
$$

The Ito form of (89) is

$$
\begin{aligned}
& d v_{x}(t)=d w_{x}(t) \\
& d v_{y}(t)=d w_{y}(t)
\end{aligned}
$$

and of course the velocity vector of the particle is a two dimensional wiener process. 
The defining relations for the flight path coordinates may be written

$$
\begin{aligned}
& v^{2}(t)=v_{x}^{2}(t)+v_{y}^{2}(t) \\
& \beta(t)=\tan ^{-1} \frac{v_{y}(t)}{v_{x}(t)}
\end{aligned}
$$

If one computes total time differentials according to the rules of ordinary calculus, one obtains

$$
\begin{aligned}
& d V=\frac{v_{x}}{V} d v_{x}+\frac{v_{y}}{v} d v_{y}=\cos \beta d v_{x}+\sin \beta d v_{y} \\
& d \beta=-\frac{v_{y}}{v^{2}} d v_{x}+\frac{v_{x}}{v^{2}} d v_{y}=-\frac{\sin \beta}{V} d v_{x}+\frac{\cos \beta}{v} d v_{y}
\end{aligned}
$$

By (91) and (93), therefore, the Stratonovich form of the stochastic equation of motion in flight path coordinates is

$$
\left[\begin{array}{l}
\alpha V(t) \\
\alpha \beta(t)
\end{array}\right]=\left[\begin{array}{cc}
\cos \beta(t) & \sin \beta(t) \\
-\frac{\sin \beta(t)}{V(t)} & \frac{\cos \beta(t)}{V(t)}
\end{array}\right]\left[\begin{array}{l}
d w_{x}(t) \\
d w_{y}(t)
\end{array}\right]
$$

Now suppose that one computes the total time differential of (92) according to the rules of the Ito stochastic calculus, or alternatively, one computes the Ito correction term for (94) according to the rule given in reference 8 . Either way, the Ito differential equation corresponding to $(94)$ is 


$$
\left[\begin{array}{c}
d V(t) \\
d \beta(t)
\end{array}\right]=\left[\begin{array}{c}
\frac{1}{V(t)} \\
0
\end{array}\right] d t+\left[\begin{array}{cc}
\cos \beta(t) & \sin \beta(t) \\
-\frac{\sin \beta(t)}{V(t)} & \frac{\cos \beta(t)}{V(t)}
\end{array}\right]\left[\begin{array}{c}
d w_{X}(t) \\
d w_{y}(t)
\end{array}\right]
$$

Thus, the $d \beta$ equation is the same in both Ito and Stratonovich forms, but the $d V$ equation differs by a term $\frac{1}{V}$ dt.

Let $\mathrm{p}$ be the transition density for the $(V, \beta)$ process. The forward partial differential equation obeyed by this density can be written down from (95) using the rule given by Doob, or it can be written down from (94) using the rule given by Stratonovich. Either way, one finds that the equation is

$$
\frac{\partial p}{\partial t}=-\frac{\partial}{\partial V}\left(\frac{1}{V} p\right)+\frac{1}{2} \frac{\partial^{2} p}{\partial v^{2}}+\frac{1}{2 v^{2}} \frac{\partial^{2} p}{\partial \beta^{2}}
$$

Equation (96) is the equation obeyed by the transition density of a two dimensional Wiener process expressed in polar coordinates, as can be verified by starting with the diffusion equation in rectangular coordinates and applying the rules for change of variables in probability densities.

Summarizing what we have so far, the stochastic differential equation of motion of a particle of unit mass whose velocity vector is a planar Wiener process are given in Cartesian coordinates by (91), in Stratonovich form in flight path coordinates by (94), and in Ito form in flight path coordinates by (95). In Cartesian coordinates, the Ito and Stratonovich forms of the equations coincide; in flight path coordinates they do not coincide. The choice of which one to use is entirely a matter of personal preference, because (91), (94), and (95) are merely three different, but equivalent, ways of describing exactly the same process. 
In (89), it was implicitly assumed that $n_{x}(t)$ and $n_{y}(t)$ are independent of $v_{x}(t)$ and $v_{y}(t)$, or stated more rigorously, in (91) the increments $d v_{x}(t)$ and $d v_{y}(t)$ are independent of $v_{x}(t)$ and $v_{y}(t)$. Physically, we have a white noise force field which is fixed in inertial coordinates, through which the particle moves. When the situation is viewed in flight path coordinates, the force on the particle appears to be correlated with the flight path angle $\beta(t)$.

Since the Stratonovich equation (94) can be manipulated according to the rules of ordinary calculus, let us re-introduce the white noise forces $n_{x}$ and $n_{y}$ and rewrite (94) in engineering fashion as

$$
\left[\begin{array}{c}
\dot{V}(t) \\
\dot{V} \dot{B}(t)
\end{array}\right]=\left[\begin{array}{cc}
\cos \beta(t) & \sin \beta(t) \\
-\sin \beta(t) & \cos \beta(t)
\end{array}\right]\left[\begin{array}{l}
n_{x}(t) \\
n_{y}(t)
\end{array}\right]
$$

Both components of this vector now have the physical dimensions of force. Let $n_{\|}$and $n_{1}$ respectively be the forces parallel and perpendicular to the flight path. Thus

$$
\left[\begin{array}{l}
\left.n\right|^{(t)} \\
n^{(t)}
\end{array}\right]=\left[\begin{array}{cc}
\cos \beta(t) & \sin \beta(t) \\
-\sin \beta(t) & \cos \beta(t)
\end{array}\right]\left[\begin{array}{l}
n_{x}(t) \\
n_{y}(t)
\end{array}\right]
$$

By definition of $n_{x}$ and $n_{y}$,

$$
E\left\{\left[\begin{array}{l}
n_{x}(t) \\
n_{y}(t)
\end{array}\right]\right\}=\left[\begin{array}{l}
0 \\
0
\end{array}\right]
$$




$$
\left.E\left\{\left[\begin{array}{l}
n_{x}(t) \\
n_{y}(t)
\end{array}\right]^{\left[n_{x}(\tau)\right.} \quad n_{y}(\tau)\right]\right\}=\delta(t-\tau)\left[\begin{array}{ll}
1 & 0 \\
0 & 1
\end{array}\right]
$$

Now consider

$$
\begin{aligned}
E\left\{\left[\begin{array}{c}
\left.n_{1}\right|^{(t)} \\
n_{\perp}(t)
\end{array}\right]\right\} & =E\left\{E\left\{\left[\begin{array}{c}
\left.n_{1}\right|^{(t)} \\
n_{\perp}(t)
\end{array}\right] \mid \beta(t)\right\}\right\} \\
& =E\left\{\left[\begin{array}{cc}
\cos \beta(t) & \sin \beta(t) \\
-\sin \beta(t) & \cos \beta(t)
\end{array}\right] E\left\{\left[\begin{array}{c}
n_{X}(t) \\
n_{y}(t)
\end{array}\right] \mid \beta(t)\right\}\right\}=\left[\begin{array}{l}
0 \\
0
\end{array}\right]
\end{aligned}
$$

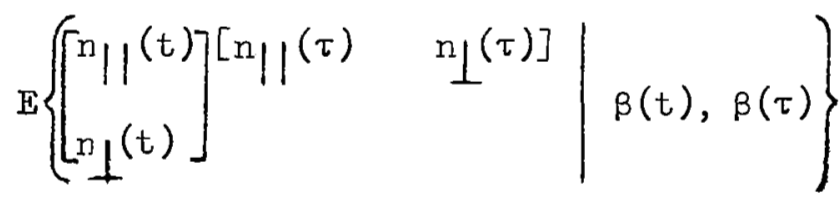

$\left.=\left[\begin{array}{cc}\cos \beta(t) & \sin \beta(t) \\ -\sin \beta(t) & \cos \beta(t)\end{array}\right] E\left\{\left[\begin{array}{ll}n_{x}(t) \\ n_{y}(t)\end{array}\right]^{\left[n_{x}(\tau)\right.} n_{y}(\tau)\right] \mid \beta(t) \beta(\tau)\right\}\left[\begin{array}{ll}\cos \beta(\tau) & -\sin \beta(\tau) \\ \sin \beta(\tau) & \cos \beta(\tau)\end{array}\right]$

$$
\begin{aligned}
& =\delta(t-\tau)\left[\begin{array}{cc}
\cos \beta(t) & \sin \beta(t) \\
-\sin \beta(t) & \cos \beta(t)
\end{array}\right]\left[\begin{array}{ll}
1 & 0 \\
0 & 1
\end{array}\right]\left[\begin{array}{cc}
\cos \beta(\tau) & -\sin \beta(\tau) \\
\sin \beta(\tau) & \cos \beta(\tau)
\end{array}\right] \\
& =\delta(t-\tau)\left[\begin{array}{cc}
\cos [\beta(t)-\beta(\tau)] & \sin [\beta(t)-\beta(\tau)] \\
-\sin [\beta(t)-\beta(\tau)] & \cos [\beta(t)-\beta(\tau)]
\end{array}\right]
\end{aligned}
$$


However, the $\delta$-function is zero except when $t=\tau$, and when $t=\tau$ the matrix in the last line in (102) becomes the identity. Thus, it appears that

$$
\left.E\left\{\left[\begin{array}{l}
n_{\|}^{(t)} \\
n \perp^{(t)}
\end{array}\right]^{\left[n f^{(\tau)}\right.} n^{(\tau)]}\right\} \beta(t), \beta(\tau)\right\}=\delta(t-\tau)\left[\begin{array}{ll}
1 & 0 \\
0 & 1
\end{array}\right]
$$

and consequently

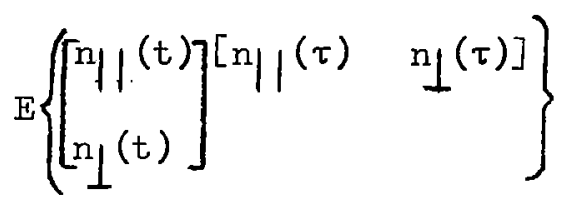

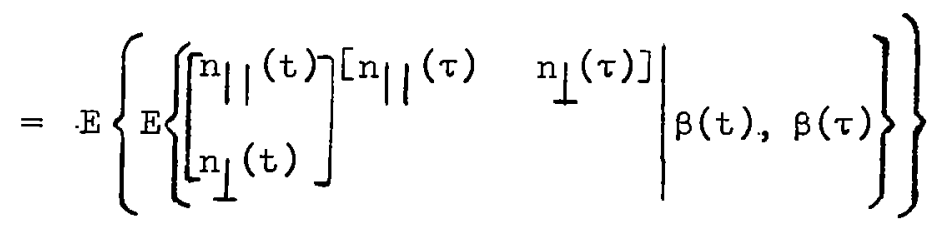

$$
\begin{aligned}
& =\delta(t-\tau)\left[\begin{array}{ll}
1 & 0 \\
0 & 1
\end{array}\right]
\end{aligned}
$$

Thus, the noise force vector $\left[\begin{array}{l}n_{1}(t) \\ n \perp(t)\end{array}\right]$ apparently has the same mean and covariance as white noise. Combining (97) and (98), one may write

$$
\left[\begin{array}{c}
\dot{v}(t) \\
v \dot{\beta}(t)
\end{array}\right]=\left[\begin{array}{l}
n_{1}(t) \\
n \\
n_{1}(t)
\end{array}\right]
$$


At first glance, (105) appears to be equivalent to what one would obtain by making (88) stochastic directly, by putting a white noise force vector on the right-hand side of (88). Let us explore this further. Let $n_{1}(t)$ and $n_{2}(t)$ be two independent Gaussian white noises, each of unity power density. Then

$$
\begin{aligned}
& E\left\{\left[\begin{array}{l}
n_{1}(t) \\
n_{2}(t)
\end{array}\right]\right\}=\left[\begin{array}{l}
0 \\
0
\end{array}\right] \\
& \left.E\left\{\left[\begin{array}{l}
n_{1}(t) \\
n_{2}(t)
\end{array}\right]^{\left[n_{1}(\tau)\right.} n_{2}(\tau)\right]\right\}=\delta(t-\tau)\left[\begin{array}{ll}
1 & 0 \\
0 & 1
\end{array}\right]
\end{aligned}
$$

Now consider the stochastic differential equation

$$
\left[\begin{array}{c}
\dot{V}(t) \\
v \dot{\beta}(t)
\end{array}\right]=\left[\begin{array}{l}
n_{1}(t) \\
n_{2}(t)
\end{array}\right]
$$

The question is, is the process generated by (108) different from the process generated by (105)? At first glance, comparing (101) to (106) and $(104)$ to (107), one is tempted to conclude that (105) and (108) generate the same process. In fact, the two processes are quite different. Introduce the two wiener processes

$$
\begin{aligned}
& w_{1}(t)=\int_{0}^{t} n_{1}(\tau) d \tau \\
& w_{2}(t)=\int_{0}^{t} n_{2}(\tau) d \tau
\end{aligned}
$$


The Ito interpretation of (108) is

$$
\left[\begin{array}{l}
d V(t) \\
d \beta(t)
\end{array}\right]=\left[\begin{array}{cc}
1 & 0 \\
0 & \frac{1}{V(t)}
\end{array}\right]\left[\begin{array}{l}
d w_{1}(t) \\
d w_{2}(t)
\end{array}\right]
$$

The forward Kolmogorov equation corresponding to (1工1) is

$$
\frac{\partial p}{\partial t}=\frac{1}{2} \frac{\partial^{2} p}{\partial v^{2}}+\frac{1}{2 v^{2}} \frac{\partial^{2} p}{\partial \beta^{2}}
$$

The two Ito equations (95) and (111) are clearly different. Further, the Kolmogorov equations (96) and (112) do not have the same solution, i.e. the transition density for the process described by (95) is different from the transition density for the process described by (lll). If two processes have different transition densities, they are different processes.

Why, then, do (105) and (108) appear to be so similar? The safest answer is that the manipulations in ( 101 ) through (104) are not only nonrigorous, but they are probably meaningless. Another answer is contained in the following plausibility argument based on the $\dot{V}$ equation alone.

For the solution $V(t)$ to (105) we have in mind exactly the same random process as the $V(t)$ component of the Ito solution of (95). Since this is the magnitude of the velocity along the flight path, it can never be negative. In fact, one can view the $\frac{1}{V(t)}$ dt Ito correction term in (95) as being the force which keeps $V(t)$ always non-negative, since the expected value of the second term in (95) is zero. Thus, $\mathrm{n} / \mid$ in (105) must somehow be correlated with $V$. 
On the other hand, for the solution $V(t)$ of (108) we have in mind exactly the same random process as the $V(t)$ component of the Ito solution of (11l). But this can be written explicitly as

$$
V(t)=V(0)+w_{1}(t)
$$

Since $w_{l}(t)$ has a Gaussian distribution, there is nothing to prevent $V(t)$ here from being negative at certain times. In fact, as soon as one realizes this, one realizes that for this reason both (108) and (III) are physically meaningless.

The main purpose of this example was to illustrate the kind of paradox one can create for oneself by trying to make direct calculations involving white noise. In any case of doubt in a modeling problem, the safe thing to do is to look at both the Ito and the Stratonovich forms of the equations, and make sure they both have a meaningful interpretation.

The ultimate objective of setting up a mathematical model is to get the predicted output of the model to be an acceptable approximation to the actually observed output of the physical system one is trying to model. This is really the only criterion by which one can judge the correctness of a model.

\section{CONCLUSION}

In this report, the problem of modeling stochastic nonlinear dynamic systems has been discussed. The various mathematical pitfalls and paradoxes that exist were illustrated by examples. It was asserted that, once the engineer understands the mathematics, he should adopt a phenomenological approach for applying it to real-world problems. 


\section{REFERENCES}

1. Wang, M. C. and Uhlenbeck, G. E., "On the Theory of the Brownian Motion II", Reviews of Modern Physics, vol. 17, pp. 323-342, 1945. Reproduced in the book, Selected Papers on Noise and Stochastic Processes, edited by N. Wax, Dover, New York, 1954.

2. Barrett, J. F., "Application of Kolmogorov's Equations to Randomly Disturbed Automatic Control Systems", Automatic and Remote Control (Proceedings of the First International Congress of IFAC), pp. 724-733, Butterworths, London, 1961.

3. Doob, J. I., Stochastic Processes, Wiley, New York, 1953.

4. Skorokhod, A. V., Studies in the Theory of Random Processes, Addison-Wesley, Reading, Mass., 1965.

5. Stratonovich, R. L., "A New Representation for Stochastic Integrals and Equations", SIAM Journal on Control, vol. 4, pp. 362-371, 1966.

6. Gray, A. H., and Caughey, T. K., "A Controversy in Problems Involving Random Parametric Excitation", Journal of Mathematics and Physics, vol. 44, No. 3, 1965, pp. 288-296.

7. McReynolds, S. R., "A New Approach to Stochastic Calculus", paper presented at the Seminar on Guidance Theory and Trajectory Analysis, INASA Electronics Research Center, May, 1967.

8. Wong, E. and Zakai, M., "On the Relation between Ordinary and Stochastic Differential Equations", International Journal of Engineering Science, vol. 3, 1965, pp. 213-229.

9. Kailath, T., "The Ito Stochastic Integral", talk presented to the Los Angeles chapter of the IEEE Professional Group on Information Theory, University of Southern California, February, 1968. 